Journal of Patient-Centered

\title{
Association of Presenting Symptoms With Abnormal Laboratory Values for Vector-Borne Illness - Experience in an Urban Gastroenterology Practice
}

\author{
Michael D. Erdman \\ Niloofar Kossari \\ Jessica Ye \\ Kristen H. Reynolds \\ Emily Blodget \\ B. Robert Mozayeni \\ Farshid Sam Rahbar
}

Follow this and additional works at: https://aah.org/jpcrr

Part of the Bacterial Infections and Mycoses Commons, Diagnosis Commons, Digestive System

Diseases Commons, Environmental Public Health Commons, Gastroenterology Commons, Infectious

Disease Commons, and the Pathological Conditions, Signs and Symptoms Commons

\section{Recommended Citation}

Erdman MD, Kossari N, Ye J, Reynolds KH, Blodget E, Mozayeni BR, Rahbar FS. Association of presenting symptoms with abnormal laboratory values for vector-borne illness - experience in an urban gastroenterology practice. J Patient Cent Res Rev. 2021;8:39-47. doi: 10.17294/2330-0698.1729

Published quarterly by Midwest-based health system Advocate Aurora Health and indexed in PubMed Central, the Journal of Patient-Centered Research and Reviews (JPCRR) is an open access, peer-reviewed medical journal focused on disseminating scholarly works devoted to improving patient-centered care practices, health outcomes, and the patient experience. 


\title{
Association of Presenting Symptoms With Abnormal Laboratory Values for Vector-Borne IIIness - Experience in an Urban Gastroenterology Practice
}

\author{
Michael D. Erdman, MBBS, ${ }^{1}$ Niloofar Kossari, MS, ${ }^{1}$ Jessica Ye, BS, ${ }^{1}$ Kristen H. Reynolds, MD, ${ }^{2}$ \\ Emily Blodget, MD, ${ }^{3}$ B. Robert Mozayeni, MD, ${ }^{4}$ Farshid Sam Rahbar, MD ${ }^{1}$ \\ ${ }^{1}$ Los Angeles Integrative Gastroenterology \& Nutrition, Los Angeles, CA; ${ }^{2}$ Aurora Wiselives Center, Advocate Aurora \\ Health, Wauwatosa, WI; ${ }^{3 K e c k}$ School of Medicine of USC, Los Angeles, CA; ${ }^{4}$ Translational Medicine Group, North \\ Bethesda, MD
}

Purpose In the clinical setting, it is not common practice to consider a vector bite, such as from a tick or flea, to be a contributing factor to chronic digestive symptoms. This article investigates associations we have observed among symptomatic patients and positive blood tests for vector-borne illness (VBI).

Methods Patients who visited an urban gastroenterology clinic over a 3-year period were retrospectively reviewed. A total of 270 patients presenting with a constellation of digestive symptoms - and who had no apparent digestive pathology and reported no prior diagnosis or treatments for VBI - were analyzed. Before the initial visit, all patients completed a review of systems medical history form, which comprised 19 gastrointestinal (GI) symptoms and 73 non-Gl-related symptoms and conditions. Patients were tested for small intestinal bacterial overgrowth (SIBO) by lactulose breath test. VBI (babesiosis, ehrlichiosis, anaplasmosis, bartonellosis, borreliosis) was established using 1 or more of several blood tests. Odds ratio (OR) analysis determined associations between exposure to VBI, SIBO, and presenting symptoms/conditions. Two age groups ( $\leq 35$ years and $\geq 36$ years) were studied using Cochran-Mantel-Haenszel stratum-based test.

Results $\quad$ A higher OR $(2.03,95 \% \mathrm{Cl}: 1.5-3.6)$ was found between patients with $\geq 3$ digestive symptoms and positive blood tests for $\geq 1 \mathrm{VBI}$. Five of the $19 \mathrm{GI}$ symptoms were independently associated with VBIpositive samples: food intolerance, indigestion, nausea/vomiting, constipation, and heartburn. A similar association in patients with $\geq 3$ non-GI symptoms (OR: 2.83, 95\% Cl: 1.3-6.4) was observed. Five of the 73 non-GI symptoms/conditions were independently associated with VBI-positive samples: chest pain, shortness of breath, extremity or joint pain, anxiety, and night sweats. Having $\geq 3$ of any digestive or nondigestive symptoms generated significant relative risk of being VBI-positive. Presence of SIBO alone did not identify significant relative risk for a VBI, and age was not a confounder.

Conclusions Findings revealed an association between positive blood tests for vector-borne illness and chronically symptomatic patients regardless of whether symptoms were digestive or nondigestive. The manifestation of 3 or more gastrointestinal and/or extraintestinal symptoms should raise suspicion for a VBI. (J Patient Cent Res Rev. 2021;8:39-47.)

Keywords bloating; functional bowel disorders; malabsorption; small intestinal bacterial overgrowth; SIBO; gastrointestinal symptoms; digestive symptoms; vector-borne illness; tick-borne; Lyme disease

$\mathrm{T}$ The human intestinal tract is composed of a complex and interactive community of 100 trillion microbial cells. ${ }^{1}$ An imbalance of the gut microbiome may have serious health consequences, ${ }^{2}$

Corresponding author: Farshid Sam Rahbar, MD, FACP, Los Angeles Integrative Gastroenterology \& Nutrition, Los Angeles, CA 90067 (drrahbar.ilads@laintegrativegi.com) including small intestinal bacterial overgrowth (SIBO). SIBO is excessive bacteria in the upper gastrointestinal (GI) tract. One may quantitatively define SIBO as an increase in the number of bacteria in the small bowel exceeding $10^{5}$ organisms $/ \mathrm{mL}$. 3 . Some authorities may consider bacterial count as high as $10^{3}$ as a threshold for having SIBO ${ }^{5}$ Common symptoms related to SIBO are abdominal bloating, distension or pain, flatulence, nausea, dyspepsia, diarrhea, constipation, fatigue, and malabsorption symptoms. ${ }^{4,6}$ 
According to the World Health Organization, vectorborne illnesses (VBI) account for more than $17 \%$ of all infectious diseases. ${ }^{7}$ In the United States, the number of reported cases of disease from mosquito, flea, and tick bites has tripled from 2004 to 2016, totaling more than 640,000 reported cases over that period. ${ }^{8}$ Recollection of a tick or vector bite is not needed to consider the diagnosis of VBI because the embedded vector/tick secretes a compound that anesthetizes the skin at the point of entry. ${ }^{9}$ Thus, most people will not feel or remember a bite. ${ }^{9}$ Ticks can infect humans with a variety of bacteria, viruses, fungi, and protozoans, all at the same time in a single bite. ${ }^{10}$

Borrelia burgdorferi sensu stricto, as well as species of the $B$. burgdorferi sensu lato complex, cause borreliosis, otherwise known as Lyme disease. Diagnosis of the acute Borrelia infection is generally made based on a subject's possible exposure to infected ticks, typical symptoms, and physical findings (eg, characteristic erythema migrans rash). Statistics reported by the U.S. Centers for Disease Control and Prevention (CDC) suggest that each year more than 300,000 individuals in the United States become infected with borreliosis, though the vast majority of these infections go unreported. ${ }^{8}$ A published survey of 3090 patients with chronic Lyme disease found that more than $50 \%$ had coinfections, with $30 \%$ reporting 2 or more coinfections. The most common coinfections were babesiosis (32\%) and bartonellosis $(28 \%) .{ }^{11}$ These coinfections with borreliosis can exacerbate symptoms or induce similar clinical manifestations. ${ }^{12}$

Babesiosis is caused by microscopic parasites, with Babesia microti being the most common species found in Americans and $B$. duncani noted mainly on the U.S. west coast. ${ }^{7}$ Babesia parasites infect and lyse red blood cells and therefore may cause hemolytic anemia in addition to nonspecific flu-like symptoms such as fever, chills, body aches, nausea, or fatigue. ${ }^{13}$ The U.S. Food and Drug Administration (FDA) has determined babesiosis to be a relevant transfusion-transmitted infection. As per FDA guidelines in July 2018, it is now recommended to screen donor blood for human babesiosis. ${ }^{14}$ This policy implies that there are many "healthy" donors who feel well enough to donate blood, yet they may be turned down because of the detection of Babesia in their bloodstream. According to the FDA, the vast majority of $B$. microti infections are asymptomatic and never diagnosed. ${ }^{14}$ In addition, the duration of $B$. microti infection in healthy adults is not clearly known, and the parasitemia may persist for more than 2 years. ${ }^{14}$ Currently, the U.S. blood screening policy does not recommend testing for B. duncani, despite its relatively wide distribution. ${ }^{9,15}$
Bartonellosis is caused by gram-negative Bartonella spp. bacteria. Common diseases caused by Bartonella are cat-scratch disease (B. henselae), Carrion's disease (B. bacilliformis), and trench fever (B. quintana). ${ }^{16}$ Symptoms range from lymphadenopathy to systemic disease, with the severity and presentation of illness related to an individual's immune status. ${ }^{17}$

The study of VBI presented herein focuses on this most prevalent trio - borreliosis, ${ }^{18}$ Bartonella spp., and Babesia spp. - along with testing for Ehrlichia and Anaplasma. We undertook this research based on the patient population that presented to our gastroenterology clinic, for whom we could not explain their persistent or recurrent digestive symptoms or difficult-to-treat SIBO. We speculated that patients with underlying VBI might present primarily with chronic digestive manifestations, leading to unfavorable SIBO treatment outcomes.

The primary goal of this retrospective study was to investigate the degree of association between positive hematological markers for VBI and the constellation of digestive and nondigestive symptoms and conditions in a patient population referred for potential SIBO, specifically, to better understand which subset of symptoms/conditions would indicate the possibility of an underlying VBI. We also evaluated the association between diagnosis of SIBO and positive hematological markers for VBI.

\section{METHODS}

A retrospective study of patients seen at an urban GI practice based in Los Angeles, California, from November 4, 2015, to October 12, 2018, was conducted under institutional review board exemption protocol (Pearl IRB no. 19-LAGA-101). First-time attendees to the practice, either self-referred or referred by another physician, were eligible for the study. Patients presented with a constellation of digestive symptoms, had no obvious GI pathology, and to the best of the practice's knowledge, had no prior diagnosis or treatments for VBI. These patients had clinical presentations and multisystemic manifestations that suggested the possibility of an underlying systemic illness.

At the initial visit, all patients completed a review of systems medical history form, which comprised 19 GI symptoms and 73 non-GI-related symptoms and conditions. The practice requested that patients circle any symptom or condition that they were experiencing. Symptomatic patients - symptoms being ones that patients circled on their own - who completed a SIBO breath test and blood sampling for VBIs after consultation with the physician were included in the study. Any 
candidate who did not complete a breath test or blood testing for VBI was excluded from the study.

\section{Methods to Determine Positive Results Small Intestinal Bacterial Overgrowth Test. For SIBO} analysis, we collected samples using the QuinTron breath test kit (QuinTron Instrument Company) and analyzed according to the protocols outlined by the 2016 SIBO consensus criteria. ${ }^{19}$ Any of the following 3 results was considered sufficient evidence of SIBO positivity: methane of $\geq 10 \mathrm{ppm}$ in the first 80 minutes; hydrogen rate of rise of $\geq 20 \mathrm{ppm}$ in the first 80 minutes; and hydrogen baseline of $\geq 20 \mathrm{ppm}$. These criteria are more stringent than the currently defined consensus for SIBO-positive results, ${ }^{19}$ placing the cutoff for the final time spot for gas measurement at 80 minutes as opposed to 90 or 120 minutes. This was done to avoid borderline cases.

Vector-Borne Illness Testing. For positive VBI, testing included Lyme immunoblot, ${ }^{20}$ general western blot, ${ }^{20}$ polymerase chain reaction (PCR), ${ }^{21}$ indirect immunofluorescent antibody, ${ }^{22}$ and fluorescent in situ hybridization (FISH) analysis. ${ }^{20}$ Detailed information about the reference clinical laboratory protocols is provided in Online Appendix A.

There are different schools of thought when it comes to clinical lab testing models for borreliosis. To reduce controversy and ambiguity, only those patients who met CDC criteria for B. burgdorferi western blot interpretation were incorporated, meaning that we excluded bands $31 \mathrm{kD}$ and $34 \mathrm{kD}$ for western blot interpretation, thereby increasing specificity. The enzyme immunoassay screening test also was excluded as a prerequisite for considering western blot results positive, out of the desire for higher sensitivity to ensure a potential association was not missed in this study, which was primarily intended to formulate a hypothesis that we hope will inform subsequent studies.

The biological rationale for these decisions was as follows: Patients with VBI are often deficient in their IgA and IgG total antibody levels. In other words, had we relied on the screening test as a prerequisite to western blot testing, we might have missed a substantial portion of this cohort and therefore any association. Additionally, in the last one-third of our study, patients were tested for borreliosis by immunoblot and did not have a western blot test. Immunoblot testing does not follow the same enzyme immunoassay first-step prerequisite.

Lyme Immunoblot. B. burgdorferi sensu lato-specific $\operatorname{IgM}$ and $\operatorname{IgG}$ antibodies were detected in human serum samples by the Lyme immunoblot test. ${ }^{20}$ Recombinant proteins to the following Borellia species - B. burgdorferi
B31, B. burgdorferi 297, B. californiensis, B. mayonii, B. spielmanii, B. afzelii, B. garinii, and B. valaisiana were sprayed at specific positions onto a nitrocellulose membrane and cut into strips. These strips were then used to detect $B$. burgdorferi sensu lato-specific antibodies in patient serum, as described by Liu et al. ${ }^{20}$ The blots were interpreted according to $\mathrm{CDC} / \mathrm{New}$ York State criteria. IgM immunoblot was deemed positive if 2 of the following 3 bands were present: 23,39 , and $41 \mathrm{kDa}$. IgG immunoblot was deemed positive if 5 of the following 10 bands were present: $18,23,28,30,39,41,45,58,66$, and $93 \mathrm{kDa}$.

Tick-Borne Relapsing Fever (TBRF) Immunoblot. This test is a qualitative immunoblot assay that detects $\operatorname{IgM}$ and IgG antibodies in human serum to the following TBRFassociated Borrelia species: B. miyamotoi, B. hermsii, B. turicatae, and B. coriaceae. Recombinant TBRF Borrelia antigens were sprayed at specific positions onto a nitrocellulose membrane and cut into strips. These strips were used to detect TBRF Borrelia-specific antibodies in patient serum as described for Lyme immunoblots..$^{23} \mathrm{TBRF}$ immunoblot for $\operatorname{IgM}$ or $\operatorname{IgG}$ was considered positive if at least 1 antigen from at least 2 of 4 groups $-70-75 \mathrm{kDa}$, GlpQ, 21-23kDA, and 41kDA - was detected.

Lyme Multiplex PCR Followed by Confirmation by DotBlot Assay. Lyme multiplex PCR is a 3-step amplified nucleic acid assay that detects specific DNA sequences of OspA plasmid and P66 of B. burgdorferi sensu stricto, B. afzeli, B. andersonii, and B. garinii in clinical specimens. ${ }^{21}$ Gene fragments were hybrid-selected with 3 biotin-labeled capture probes (Online Appendix A). Probe $\mathrm{A}$ and Probe $\mathrm{B}$ were designed to selectively bind OspA and Probe $\mathrm{C}$ to $\mathrm{P} 66$ gene sequences, respectively, that are conserved in different $B$. burgdorferi strains. The hybridselected $B$. burgdorferi-specific DNA fragments were captured on to streptavidin beads and washed to remove debris and excess probes. The purified bound DNA was eluted and amplified using 2 sets of primers, an OspA set described by Mouristen et $\mathrm{al}^{24}$ and a P66 gene-specific set described by Rosa et $\mathrm{a}^{25}$ (Online Appendix A). The amplified PCR products were detected in a final dot-blot assay by two $B$. burgdorferi-specific $5^{\prime}$ digoxigeninlabeled probes using the Roche hybridization kit (Roche Molecular Diagnostics). The first probe targeted the genomic flagellin gene, and the second probe targeted the plasmid-encoded $O s p A$ gene (Online Appendix A). A sample is considered positive if the dot-blot is positive.

Indirect Immunofluorescent Antibody. Respective indirect immunofluorescent antibody tests detect IgM and IgG antibodies to Babesia species B. microti, B. duncani, and $B$. henselae in human serum. The human monocytic ehrlichiosis immunofluorescent antibody test detects IgM 
and IgG antibodies to Ehrlichia chaffeensis, the causative agent of ehrlichiosis in human serum. The human granulocytic anaplasmosis immunofluorescent antibody test is used to detect IgM and IgG antibodies to Anaplasma phagocytophilum, the causative agent of anaplasmosis in human serum. A patient sample is considered positive if the titer is $\geq 20$ for $\operatorname{IgM}$ and $\geq 40$ for IgG.

Fluorescent In Situ Hybridization. The ribosomal RNA of Babesia (ring or merozoite form) was directly detected in a blood smear by FISH assay using Babesiaspecific DNA probes labeled with fluorescent dyes. ${ }^{26}$ The sample was considered positive if fluorescing rings or merozoites are present inside at least 2 red blood cells (RBCs). This test has excellent sensitivity and specificity over standard Giemsa-stained smears for the presence of intraerythrocytic parasites (piroplasts) in red blood cells. ${ }^{26}$ The Bartonella FISH test, which is similar to FISH for Babesia, also was performed on ethylenediaminetetraacetic-acid whole blood smears. The sample was considered positive if fluorescing rodshaped bodies were detected in the smear. ${ }^{23}$ The FISH test detects bacteria of the genus Bartonella, including $B$. vinsonii, $B$. berkhoffii, $B$. henselae, and $B$. quintana, and provides a significant increase in specificity over the standard gram stain for the presence of Bartonella in whole blood smears. ${ }^{23}$

\section{Statistical Analysis}

Associations between symptoms and test results were quantified using odds ratios (OR) and their 95\% confidence intervals $(\mathrm{CI})$. We analyzed the confounding effect of age using the Cochran-Mantel-Haenszel method.

\section{RESULTS}

A total of 270 patients seen from November 4, 2015, to October 12, 2018, met the criteria for our retrospective study. Selected patients presented with a variety of digestive (ie, GI) symptoms and nondigestive (ie, nonGI) symptoms/conditions and were found to have SIBO $(n=173)$ or not have SIBO $(n=97)$ and have VBI $(n=136)$ or not have VBI $(n=134)$. Patient demographics are outlined in Table 1.

\section{Association Between SIBO and VBI}

Figure 1 shows that out of 270 patients, 173 patients had SIBO. When comparing the SIBO-positive and SIBO-negative groups, both showed approximately 50\% prevalence for VBI. The calculated OR of $0.99(95 \%$ CI: 0.6027-1.6294) negated a clear cause-and-effect association between VBI and SIBO, meaning that patients with evidence of at least 1 positive blood test for a VBI were no more likely to have SIBO than patients who had negative VBI blood tests.

\section{Composition of GI Symptoms and Association With VBI}

We studied 19 GI symptoms that patients circled on their first-visit intake form. As shown in Table 2, 5 of the 19 GI symptoms had significantly higher odds when determining an association with VBI-positive blood samples (OR range: 1.65-2.01). These 5 symptoms were food intolerance, indigestion, nausea/vomiting, constipation, and heartburn.

Table 3 highlights the importance of the number of GI symptoms when determining an association with positive blood tests for VBI. Having 3 or more of any of the 19 GI symptoms was associated with positive VBI.

Table 1. Demographic Features of Patients $(N=270)$

\begin{tabular}{lc}
\hline Demographic & $\mathbf{n}$ \\
\hline Female, $\mathrm{n}$ & 170 \\
Male, $\mathrm{n}$ & 100 \\
Age in years, median (range) & $39(13-76)$ \\
SIBO-positive, $\mathrm{n}$ & 173 \\
SIBO-negative, $\mathrm{n}$ & 97 \\
VBI-positive, $\mathrm{n}$ & 136 \\
VBI-negative, $\mathrm{n}$ & 134 \\
Age of $\leq 35$ years, $\mathrm{n}$ & 110 \\
Age of $\geq 36$ years, $\mathrm{n}$ & 160 \\
Age of $\leq 35$ years with VBI, $\mathrm{n}$ & 59 \\
Age of $\geq 36$ years with VBI, $\mathrm{n}$ & 77 \\
\hline
\end{tabular}

SIBO, small intestinal bacterial overgrowth; VBI, vectorborne illness.

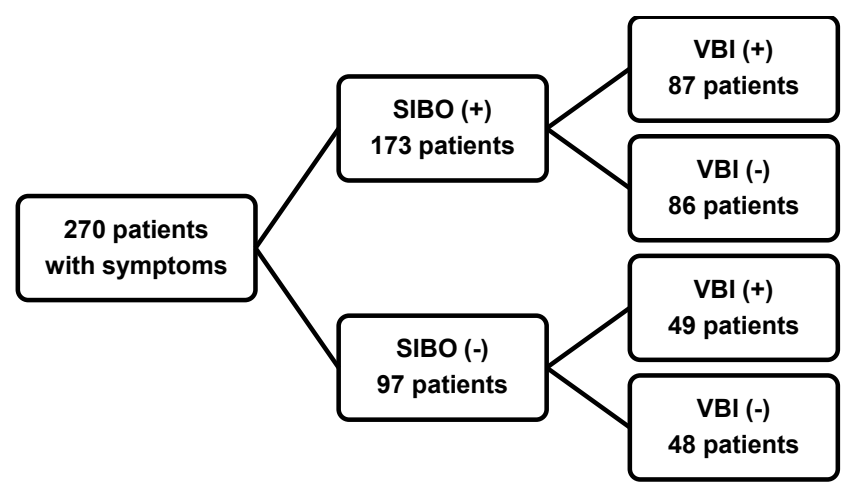

Figure 1. Prevalence of blood test-verified vectorborne illness (VBI) in patients with and without small intestinal bacterial overgrowth (SIBO). Analysis comparing all VBI subgroups yielded an odds ratio of 0.99 (95\% Cl: 0.6027-1.6294). SIBO (+) stands for SIBO-positive; SIBO (-), SIBO-negative; VBI (+), VBIpositive; and VBI (-), VBI-negative. 
Table 2. Associations Between 19 Individual Digestive Symptoms and Positive Blood Tests for Vector-Borne Illness in a Retrospectively Reviewed 270-Patient Sample

\begin{tabular}{l|cc|cc|cc}
\hline & \multicolumn{2}{|c|}{ VBI (n=136) } & \multicolumn{2}{c|}{ Non-VBI (n=134) } & \\
\cline { 2 - 5 } Gastrointestinal symptom & Case & Control & Case & Control & Odds ratio & 95\% Cl \\
\hline Abdomen pain & 75 & 61 & 70 & 64 & 1.124 & $0.6964-1.8142$ \\
Acidic stomach & 42 & 94 & 33 & 101 & 1.3675 & $0.8005-2.3362$ \\
Altered bowel & 52 & 84 & 39 & 95 & 1.5079 & $0.9069-2.5073$ \\
Bloating & 111 & 25 & 101 & 33 & 1.4507 & $0.8078-2.6051$ \\
Constipation & 62 & 74 & 40 & 94 & 1.9689 & $1.1933-3.2487$ \\
Dark urine & 9 & 127 & 3 & 131 & 3.0945 & $0.8190-11.6918$ \\
Diarrhea & 47 & 89 & 32 & 102 & 1.6833 & $0.9892-2.8644$ \\
Difficulty swallowing & 15 & 121 & 12 & 122 & 1.2603 & $0.5665-2.8040$ \\
Feeding difficulty & 6 & 130 & 2 & 132 & 3.0462 & $0.6037-15.3703$ \\
Food intolerance & 63 & 73 & 46 & 88 & 1.651 & $1.0107-2.6968$ \\
Heartburn & 37 & 99 & 21 & 113 & 2.0111 & $1.1041-3.6631$ \\
Indigestion & 53 & 83 & 36 & 98 & 1.7383 & $1.0392-2.9077$ \\
Irregular bowel movements & 48 & 88 & 40 & 94 & 1.28 & $0.7694-2.1356$ \\
Lack of appetite & 30 & 106 & 18 & 116 & 1.8239 & $0.96-3.4623$ \\
Nausea vomiting & 48 & 88 & 31 & 103 & 1.8123 & $1.0627-3.0906$ \\
Need for laxatives & 15 & 121 & 11 & 123 & 1.3862 & $0.6120-3.1395$ \\
Rectal bleeding & 12 & 124 & 9 & 125 & 1.3441 & $0.5469-3.3035$ \\
Weight loss & 31 & 105 & 23 & 111 & 1.4248 & $0.7806-2.6009$ \\
Weight gain & 24 & 112 & 22 & 112 & 1.0909 & $0.5781-2.0587$ \\
\hline
\end{tabular}

In this table, "Case" refers to patients who were positive for the symptom. "Control" refers to patients who were negative for the symptom. "VBI" refers to patients with positive blood tests for vector-borne illness. "Non-VBI" refers to patients with negative blood tests for vector-borne illness.

Table 3. Associations Between Number of Presenting Gastrointestinal (GI) Symptoms and Blood Test Results for Vector-Borne Illness in a Retrospectively Reviewed 270-Patient Sample

\begin{tabular}{|c|c|c|c|c|c|c|}
\hline \multirow[b]{2}{*}{ Gl symptoms } & \multicolumn{2}{|c|}{ VBI $(n=136)$} & \multicolumn{2}{|c|}{ Non-VBI $(n=134)$} & \multirow[b]{2}{*}{ Odds ratio } & \multirow[b]{2}{*}{$95 \% \mathrm{Cl}$} \\
\hline & Case & Control & Case & Control & & \\
\hline 0 or more & 136 & 0 & 134 & 0 & 1.0149 & $0.0200-51.5209$ \\
\hline 1 or more & 125 & 11 & 126 & 8 & 0.7215 & $0.2808-1.8540$ \\
\hline 2 or more & 119 & 17 & 113 & 21 & 1.3009 & $0.6529-2.5919$ \\
\hline 3 or more & 111 & 25 & 92 & 42 & 2.027 & $1.1497-3.5735$ \\
\hline 4 or more & 100 & 36 & 72 & 62 & 2.392 & $1.4359-3.9847$ \\
\hline 5 or more & 88 & 48 & 60 & 74 & 2.2611 & $1.3859-3.6891$ \\
\hline 6 or more & 70 & 66 & 42 & 92 & 2.3232 & $1.4143-3.8162$ \\
\hline 7 or more & 53 & 83 & 27 & 107 & 2.5306 & $1.4674-4.3639$ \\
\hline
\end{tabular}

In this table, "Case" refers to patients with Gl symptoms that met the specific parameter criteria. "Control" refers to patients with Gl symptoms that did not meet specific parameter criteria. "VBl" refers to patients with positive serology for vector-borne illness. "Non-VBl" refers to patients with negative serology for vector-borne illness.

\section{Composition of Non-GI Symptoms and Association With VBI}

We studied 73 non-GI symptoms/conditions that patients circled on their first-visit intake form. As shown in Table 4, 5 symptoms independently had higher odds when determining an association with
VBI-positive blood samples (OR range: 1.79-2.32). These 5 non-GI symptoms included extremity and joint pain, chest pain, shortness of breath, anxiety, and night sweats. For a complete table of the 73 non-GI symptoms/conditions with their respective OR, see Online Appendix B. 
Table 4. Five Independent Nondigestive Symptoms That Demonstrated an Association With Positive Blood Tests for Vector-Borne Illness in a Retrospectively Reviewed 270-Patient Sample

\begin{tabular}{|c|c|c|c|c|c|c|}
\hline \multirow[b]{2}{*}{ Symptom } & \multicolumn{2}{|c|}{ VBI $(n=136)$} & \multicolumn{2}{|c|}{ Non-VBI $(n=134)$} & \multirow[b]{2}{*}{ Odds ratio } & \multirow[b]{2}{*}{$95 \% \mathrm{Cl}$} \\
\hline & Case & Control & Case & Control & & \\
\hline Extremity and joint pain & 43 & 93 & 27 & 107 & 1.8323 & $1.0511-3.1941$ \\
\hline Chest pain & 29 & 107 & 14 & 120 & 2.3231 & $1.1663-4.6272$ \\
\hline Shortness of breath/Air hunger & 37 & 99 & 19 & 115 & 2.2621 & $1.2230-4.1841$ \\
\hline Anxiety & 90 & 46 & 70 & 64 & 1.7888 & $1.0948-2.9229$ \\
\hline Night sweats & 34 & 102 & 19 & 115 & 2.0175 & $1.0837-3.7560$ \\
\hline
\end{tabular}

In this table, "Case" refers to patients who were positive for the symptom. "Control" refers to patients who were negative for the symptom. "VBI" refers to patients with positive blood tests for vector-borne illness. "Non-VBI" refers to patients with negative blood tests for vector-borne illness.

Table 5. Associations Between Number of Presenting Nongastrointestinal (Non-GI) Symptoms and Blood Test Results for Vector-Borne Illness in a Retrospectively Reviewed 270-Patient Sample

\begin{tabular}{l|cc|cc|cc}
\hline \multirow{2}{*}{ Non-GI symptoms } & \multicolumn{2}{|c|}{ VBI $(\mathbf{n = 1 3 6 )}$} & \multicolumn{2}{|c|}{ Non-VBI $(\mathbf{n = 1 3 4 )}$} & \multirow{2}{*}{ Odds ratio } & $95 \%$ Cl \\
\cline { 2 - 5 } 1 or more & Case & Control & Case & Control & Odds \\
2 or more & 108 & 28 & 97 & 37 & 1.4713 & $0.8385-2.5817$ \\
3 or more & 54 & 82 & 40 & 94 & 1.5476 & $0.9341-2.5640$ \\
\hline
\end{tabular}

In this table, "Case" refers to patients with non-Gl symptoms that met the specific parameter criteria. "Control" refers to patients with non-GI symptoms that did not meet specific parameter criteria. "VBI" refers to patients with positive blood tests for vector-borne illness. "Non-VBI" refers to patients with negative blood tests for vector-borne illness.

Table 5 indicates the importance of the number of non-GI symptoms when determining an association with positive blood tests for VBI. It was only when 3 or more of any of the 73 non-GI symptoms/conditions were present that an association with VBI testing was detected.

Table 6 depicts detailed breakdown of multiple parameters shown on the left, and the positive blood test results of the 9 different groups of infections (Lyme, TBRF group, $B$. microti, $B$. dancani, human monocytic ehrlichiosis, human granulocytic anaplasmosis, $B$. henselae, Rickettsia rickettsii, and Rickettsia typhi). Of interest, positive blood markers for B. duncani were most prevalent in every parameter studied.

The Cochran-Mantel-Haenszel statistical test was used to study age as a possible confounder. According to the test data in patients who were $\leq 35$ years old versus those who were $\geq 36$ years old, age was not a confounding factor $\left(\chi^{2}\right.$ : 10.26, OR: 2.5 , 95\% CI: $1.45-4.3)$. Gender also did not appear to be a confounding factor.

\section{DISCUSSION}

In the setting of a face-to-face evaluation of a patient in the exam room, it is uncommon to consider a vector bite, such as one from a tick or flea, to be the primary contributor to digestive symptoms and chronic illness. However, our experience suggests the contrary. In our study population, patients presented with chronic GI symptoms and evidence of positive laboratory markers for VBI and yet did not have classic acute presentation.

Tick and/or other vector-borne diseases are generally recognized based on their acute signs - a scenario not typically encountered in gastroenterology practices. The history of medicine, however, reveals that many of the diseases caused by other unrelated microbial agents may indeed become persistent without a patient's recollection of an acute illness. Examples of these include tuberculosis, toxoplasmosis, Zika virus, syphilis, and more. ${ }^{27-30}$

One example of a microbe that may be chronically present in the human body, with or without symptoms, is Babesia. In our own study observation, $B$. duncani was the most common pathogen identified by blood test analysis. As noted in the introduction, blood bank screening currently includes $B$. microti only, which implies that those infected with $B$. duncani might not be identified. 


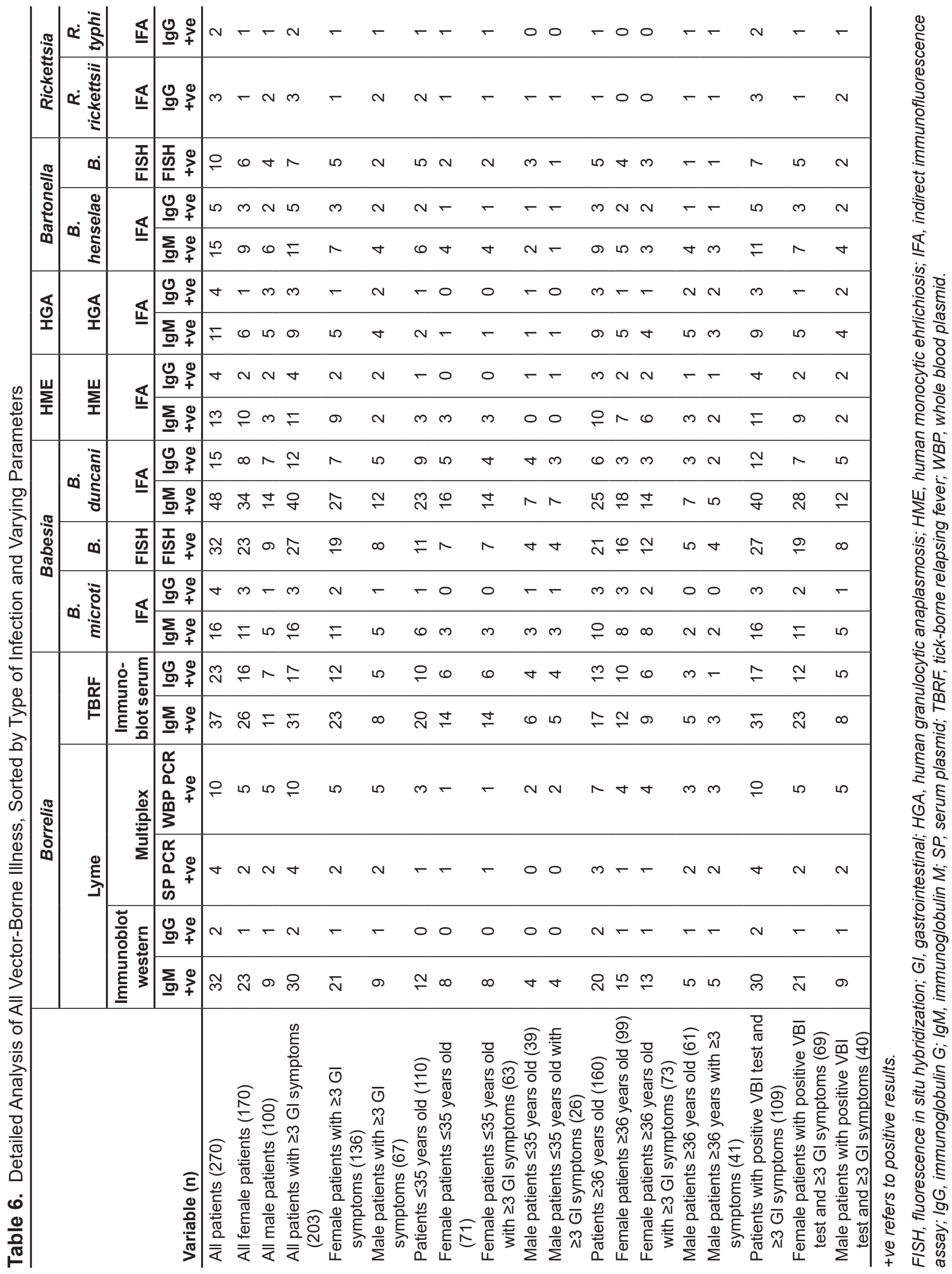


Another example is the concept of "chronic Lyme disease," which has been an area of ongoing controversy and scientific debate. A case definition for chronic Lyme disease, with supportive literature, has been proposed as a multisystem illness with a wide range of symptoms that are either continuously or intermittently present for a minimum of 6 months. ${ }^{12}$ These concepts should point to the consideration that "stealth" or persistent infections are a reality and may underlie many human maladies.

Our data suggest that one should consider an underlying microbial etiology for chronic or unexplained digestive conditions that do not resolve. It is well described in the literature that microbial involvement may lead to immune dysregulation, ${ }^{31}$ such as Th1, Th2, ${ }^{32}$ Treg, ${ }^{33}$ and Th17 imbalances, ${ }^{34}$ mast cell activation, ${ }^{35}$ and dysautonomia, ${ }^{36}$ to name a few. The clinical manifestation often surfaces as a result of epigenetic factors, exposure to life stressors, alcohol, environmental toxins, and overall allostatic load.

Positive blood testing for VBI may suggest prior exposure to a specific organism as opposed to an active infection. The argument would then be that the clinical picture is dictated by either an infection or as a consequence of prior exposure with subsequent immune dysregulation.

\section{Limitations}

Patient age (ie, being younger $[\leq 35$ years] or older $[\geq 36$ years]) was not determined to be a confounding factor. ${ }^{37}$ Possibly there are other confounders, such as gender, height, weight, and zip code, that we did not fully analyze - a limitation of the study. We hope this article will function as a prompt to further prospective studies that factor in such potential confounders. This retrospective study has other limitations. For one, symptoms were not quantified. In addition, given the nature of our practice, we may have received a skewed population of patients and findings may not be generalizable to other practices. Finally, SIBO appears to be common in patients with positive markers for VBI; however, we found that SIBO was equally prevalent in those without VBI. Considering that we used diagnostic criteria more stringent for detecting SIBO than currently recommended guidelines, it is possible that the association was missed.

\section{Future Research}

Bearing in mind the limitations of our study, we believe that there is still value in the information provided, as our clinical and statistical findings suggest that underlying VBI may contribute to the etiology of GI symptoms. Because cause-and-effect cannot be established based on this study, we believe this hypothesis warrants further research. We propose that while many patients with digestive symptoms may strictly have a GI disease, one should at least consider the possibility of an unidentified VBI dysregulating the immune system ${ }^{38,39}$ and contributing to ongoing illness.

\section{CONCLUSIONS}

Pattern recognition is the key principle in making a proper diagnosis. In evaluating patients with chronic, nonspecific, recurrent, or unexplained digestive symptoms, health care providers should incorporate a detailed review of systems. The manifestation of 3 or more gastrointestinal symptoms and/or 3 or more extraintestinal symptoms should raise suspicion for a vector-borne illness. Recognizing underlying VBI may improve our understanding of persistent infections, resultant immune dysregulation, and their contribution to chronic, complex illness. This article can ignite additional research in this area.

\section{Patient-Friendly Recap}

- Disease caused by insect or tick bites, referred to as vector-borne illness, often goes undiagnosed. Similarly, many patients with irritiable bowel-type symptoms actually have SIBO, or small intestinal bacterial overgrowth.

- The authors studied whether patients seen for chronic unexplained symptoms were more likely to have underlying vector-borne illness and whether such illness was associated with a SIBO diagnosis.

- They found that while many in this patient population did indeed have SIBO, vector-borne illness was no more common in those who did than those who didn't. Importantly, presence of 3 or more chronic symptoms of any kind should raise suspicion for vector-borne diseases.

\section{Acknowledgments}

The authors acknowledge the clinical laboratory IGeneX Inc. (Milpitas, CA), Dr. Mehrdad Tajkarimi of Arya Technologies (Greensboro, NC), and Andrea Vasquez, operations manager, and Romtin Rahbar, fund raising and edits, both of Los Angeles Integrative Gastroenterology \& Nutrition.

\section{Author Contributions}

Study design: Erdman, Rahbar. Data acquisition or analysis: Erdman. Manuscript drafting: Erdman, Kossari, Ye. Critical revision: Erdman, Reynolds, Blodget, Mozayeni, Rahbar.

\section{Conflicts of Interest}

None.

\section{Funding Sources}

This work was supported by Patients Helping Patients, Inc. (Los Angeles, CA), a nonprofit corporation (501c3) founded by coauthor Farshid Sam Rahbar. 


\section{References}

1. Grace E, Shaw C, Whelan K, Andreyev HJN. Review article: small intestinal bacterial overgrowth - prevalence, clinical features, current and developing diagnostic tests, and treatment. Aliment Pharmacol Ther. 2013;38:674-88. CrossRef

2. Guinane CM, Cotter PD. Role of the gut microbiota in health and chronic gastrointestinal disease: understanding a hidden metabolic organ. Therap Adv Gastroenterol. 2013;6:295-308. CrossRef

3. Dukowicz AC, Lacy BE, Levine GM. Small intestinal bacterial overgrowth: a comprehensive review. Gastroenterol Hepatol (N Y). 2007;3:112-22.

4. Krajicek EJ, Hansel SL. Small intestinal bacterial overgrowth: a primary care review. Mayo Clin Proc. 2016;91:1828-33. CrossRef

5. Rao SS, Bhagatwala J. Small intestinal bacterial overgrowth: clinical features and therapeutic management. Clin Transl Gastroenterol. 2019;10:e0078. CrossRef

6. Pimentel M. Breath testing for small intestinal bacterial overgrowth: Should we bother? Am J Gastroenterol. 2016;111:307-8. CrossRef

7. World Health Organization. Vector-borne disease. Accessed December 1, 2020. https://www.who.int/news-room/factsheets/detail/vector-borne-diseases

8. Centers for Disease Control and Prevention. Illnesses from mosquito, tick, and flea bites increasing in the US. Press release issued May 1, 2018; accessed June 19, 2020. https://www.cdc. gov/media/releases/2018/p0501-vs-vector-borne.html

9. Scott JD, Scott CM. Human babesiosis caused by Babesia duncani has widespread distribution across Canada. Healthcare (Basel). 2018;6(2):49. CrossRef

10. Brites-Neto J, Duarte KM, Martins TF. Tick-borne infections in human and animal population worldwide. Vet World. 2015;8:301-15. CrossRef

11. Johnson L, Wilcox S, Mankoff J, Stricker RB. Severity of chronic Lyme disease compared to other chronic conditions: a quality of life survey. PeerJ. 2014;2:e322. CrossRef

12. Berghoff W. Chronic Lyme disease and co-infections: differential diagnosis. Open Neurol J. 2012;6:158-78. CrossRef

13. Centers for Disease Control and Prevention. Parasites - babesiosis. National surveilance. Page reviewed May 18, 2018; accessed June 19, 2020. https://www.cdc.gov/parasites/babesiosis/surveillance.html

14. U.S. Food and Drug Administration. Recommendations for reducing the risk of transfusion-transmitted babesiosis: guidance for industry. Posted May 2019; accessed June 19, 2020. https://www.fda.gov/media/114847/download

15. Scott JD. First record of locally acquired human babesiosis in Canada caused by Babesia duncani: a case report. SAGE Open Med Case Rep. 2017;5:2050313X17725645. CrossRef

16. Centers for Disease Control and Prevention. Bartonella infection (cat scratch disease trench fever, and, Carrión's disease). Accessed December 1, 2020 https://www.cdc.gov/bartonella/index.html

17. Anderson BE, Neuman MA. Bartonella spp. as emerging human pathogens. Clin Microbiol Rev. 1997;10:203-19. CrossRef

18. Centers for Disease Control and Prevention. Lyme disease. Page last reviewed November 5, 2020; accessed December 1, 2020. https://www.cdc.gov/lyme/index.html

19. Rezaie A, Buresi M, Lembo A, et al. Hydrogen and methane-based breath testing in gastrointestinal disorders: the North American Consensus. Am J Gastroenterol. 2017;112:775-84. CrossRef

20. Liu S, Cruz ID, Ramos CC, Taleon P, Ramasamy R, Shah J. Pilot study of immunoblots with recombinant Borrelia burgdorferi antigens for laboratory diagnosis of Lyme disease. Healthcare (Basel). 2018;6(3):99. CrossRef
21. Shah JS, Cruz ID, Ward S, Harris NS, Ramasamy R. Development of a sensitive PCR-dot blot assay to supplement serological tests for diagnosing Lyme disease. Eur J Clin Microbiol Infect Dis. 2018;37:701-9. CrossRef

22. Marques AR. Revisiting the Lyme disease serodiagnostic algorithm: the momentum gathers. $J$ Clin Microbiol. 2018;56(8):e00749-18. CrossRef

23. IGeneX Inc. Interpreting test results: TBRF and B. burgdorferi sensu lato real-time PCR. Accessed December 1, 2020. https:// igenex.com/test-interpretations/\#drawer-7

24. Mouritsen CL, Wittwer CT, Litwin CM, et al. Polymerase chain reaction detection of Lyme disease: correlation with clinical manifestations and serologic responses. Am J Clin Pathol. 1996;105:647-54. CrossRef

25. Rosa PA, Schwan TG. A specific and sensitive assay for the Lyme disease spirochete Borrelia burgdorferi using the polymerase chain reaction. J Infect Dis. 1989;160:1018-29. C CrossRef

26. Shah JS, Horowitz R, Harris NS. Human babesiosis and ehrlichiosis - current status. Eur Infect Dis. 2012;6:49-56

27. Teale A, Payne M, England J, Morshed M, Hull M. Zika virus, an emerging flavivirus, as a cause of fever and rash in a traveller returning from Central America. Can Commun Dis Rep. 2016;42:68-71. CrossRef

28. Flynn JL, Chan J. Tuberculosis: latency and reactivation. Infect Immun. 2001;69:4195-201. CrossRef

29. Flegr J, Prandota J, Sovičková M, Israili ZH. Toxoplasmosis - a global threat. Correlation of latent toxoplasmosis with specific disease burden in a set of 88 countries. PLoS One. 2014;9(3):e90203 CrossRef

30. Dhanaselvi H, Kalaivani S. Untreated late latent syphilis of both spouses with observation of Kassowitz law: adverse pregnancy outcomes in the postpenicillin era. Indian $J$ Dermatol. 2017;62:221-2. CrossRef

31. Lobo LA, Benjamim CF, Oliveira AC. The interplay between microbiota and inflammation: lessons from peritonitis and sepsis. Clin Transl Immunology. 2016;5(7):e90. CrossRef

32. Weinberg ED. Iron loading: a risk factor for Whipple's disease? Med Hypotheses. 2001;57:59-60. CrossRef

33. Narayana JL, Huang $\mathrm{HN}, \mathrm{Wu} \mathrm{CJ}$, Chen JY. Efficacy of the antimicrobial peptide TP4 against Helicobacter pylori infection: in vitro membrane perturbation via micellization and in vivo suppression of host immune responses in a mouse model. Oncotarget. 2015;6:12936-54. CrossRef

34. Vorob'ev AA, Bykovskaia SN, Pashkov EP, Bykov AS. [The role of regulatory cells CD4+CD25+ in the development of chronic infective diseases]. Vestn Ross Akad Med Nauk. 2006;(9-10):24-9.

35. Kim SH, Jun CD, Suk K, et al. Gallic acid inhibits histamine release and pro-inflammatory cytokine production in mast cells. Toxicol Sci. 2006;91:123-31. CrossRef

36. Mckeon A, Benarroch EE. Autoimmune autonomic disorders. Handb Clin Neurol. 2016;133:405-16. CrossRef

37. Gansky SA, Cheng NF, Koch GG. Dose-weighted adjusted Mantel-Haenszel tests for numeric scaled strata in a randomized trial. Stat Biopharm Res. 2011;3:266-75. CrossRef

38. Lochhead RB, Strle K, Kim ND, et al. MicroRNA expression shows inflammatory dysregulation and tumor-like proliferative responses in joints of patients with postinfectious Lyme arthritis. Arthritis Rheumatol. 2017;69:1100-10. CrossRef

39. Iliopoulou BP, Huber BT. Infectious arthritis and immune dysregulation: lessons from Lyme disease. Curr Opin Rheumatol. 2010;22:451-5. CrossRef

(C) 2021 Advocate Aurora Health, Inc. 